\title{
Application of Quick Response Code in Sea Turtle Conservation: Overcoming Limitations of Existing Individual Markers
}

\author{
Zhongrong Xia' ${ }^{1}$, Haoqi $\mathrm{Yu}^{2}$, Fan Yang ${ }^{3}$, Xiaofeng Liu${ }^{4}$, Dongmei $\mathrm{Yu}^{2 *}$ and Huijian $\mathrm{Hu}^{2 *}$ \\ 1 Guangdong Huidong Sea Turtle National Nature Reserve Bureau, Huizhou, China, ${ }^{2}$ Guangdong Key Laboratory of Animal \\ Conservation and Resource Utilization, Guangdong Public Laboratory of Wild Animal Conservation and Utilization, Institute \\ of Zoology, Guangdong Academy of Sciences, Guangzhou, China, ${ }^{3}$ Guangzhou Linmao Natural Technology Co., Ltd., \\ Guangzhou, China, ${ }^{4}$ College of Internet of Things Engineering, Hohai University, Changzhou, China
}

OPEN ACCESS

Edited by:

Wenguang Liu,

South China Sea Institute

of Oceanology, Chinese Academy

of Sciences (CAS), China

Reviewed by:

Min Li,

South China Sea Fisheries Research Institute, Chinese Academy of Fishery

Sciences (CAFS), China

Weijun Wang,

Ludong University, China

*Correspondence:

Dongmei Yu yudongmei50@163.com

Huijian $\mathrm{Hu}$

13922339577@139.com

Specialty section:

This article was submitted to

Marine Fisheries, Aquaculture

and Living Resources,

a section of the journal

Frontiers in Marine Science

Received: 19 November 2021

Accepted: 21 December 2021

Published: 07 February 2022

Citation:

Xia Z, Yu H, Yang F, Liu X, Yu D and $H u H$ (2022) Application of Quick

Response Code in Sea Turtle Conservation: Overcoming Limitations

of Existing Individual Markers.

Front. Mar. Sci. 8:818185.

doi: $10.3389 /$ fmars.2021.818185
Hatchery release has been used for the conservation of green turtles for many years. To improve the rescue management of released individuals and avoid their negative impact on wild populations, the application of individual markers is particularly important. Quick response (QR) code was used to tag the individual of released turtles in Huidong Sea Turtle National Nature Reserve, the unique spawning site and nature reserve for sea turtles in China. The viscosity differences of four safe and non-toxic glues (e.g., epoxy resin, acrylic glue, ABS glue, and nail gel 401) in different age groups were tested. The results revealed that epoxy resin glue has the best adhesion effect on 8-year-old turtles, and the intact rate after 50 days was $100 \%$. It is suitable to be used for the rescue of stranded turtles within 50 days of release. Nail gel 401 has the best adhesion effect on the carapace of 1 -year-old turtles, with an intact rate of $100 \%$ after 50 days, which can be used for short-time conservation of young turtles. The release experiments of 18 eight-year-old sea turtles with passive integrated transponder (PIT) tags, QR codes, and steel tags showed that QR codes are conducive to public participation and improve rescue efficiency. In fact, QR code is a powerful measure to overcome the limitations of the existing individual label. However, the QR code fused with turtle carapace can only be applied for turtle rescue and tracing in the short-term due to its limited persistence.

Keywords: QR code, sea turtle, individual identification, rescue, conservation

\section{INTRODUCTION}

There are seven existing species of sea turtles, including loggerhead (Caretta caretta), hawksbill (Eretmochelys imbricata), olive ridley (Lepidochelys olivacea), Kemp's ridley (Lepidochelys kempii), green turtle (Chelonia mydas), flatback (Natator depressus), and leatherback (Dermochelys coriacea). However, despite their international protection and conservation status, they are highly threatened by climate change, illegal hunting, and incidental fisheries by catch. The 2014 International Union for Conservation of Nature (IUCN) Red List classified all sea turtle species (excluding flatback with insufficient data) as vulnerable, endangered, or critically endangered (Vilaça et al., 2021), and their global and local population size is decreasing. 
There are five species of sea turtles (i.e., loggerhead, hawksbill, olive ridley, green turtle, and leatherback) in China, of which the population of green sea turtles is the largest. Huidong Sea Turtle National Nature Reserve is the unique spawning site and nature reserve of sea turtles in China. The bureau is responsible for the scientific research and management of sea turtle treatment, breeding, and hatchery release. In fisheries and wildlife management, hatchery release is one of the most popular stock enhancement measures. It has been used for the conservation of green sea turtles for more than 10 years. So far, of more than 1,400 natural spawnings protected in Huidong Sea Turtle National Nature Reserve, at least 90,000 eggs have been hatched, and more than 60,000 green sea turtles have been released to the South China Sea to increase the wild population.

Population enhancement of endangered animals refers to the release of endangered animals from hatcheries into natural habitats where animal populations already exist to restore populations and natural recruitment of threatened and endangered species (Katalinas et al., 2018; Loneragan et al., 2019). Such practice is frequently used worldwide and shows common success (Ueda, 2018; Kitada, 2018, 2020). To improve the rescue management of these released individuals and avoid their negative impacts on wild populations, the application of individual markers is particularly important. For turtles, commonly used individual tags include traditional external tags and advanced internal tags, such as metal tags, plastic tags, spraying tags, passive integrated transponder (PIT) tags, radio frequency identification (RFID), and satellite telemetry. Traditional external tags (e.g., metal tags, plastic tags, and spraying tags) have many disadvantages, especially loss or illegibility of tags, and the tags may alter the physiology and behavior of the tagged individuals. Advanced internal tags (e.g., PIT, RFID, and satellite telemetry) are difficult to achieve public participation because of their expensive cost and dependence on the professional operation. Two or three tags are often used together to complement each other and improve practicability. However, the abovementioned tags are less conductive to the rescue of sea turtles due to the lack of convenience for public communication.

To address this issue, we attempted to develop a simple, flexible, and inexpensive platform to generate and store electronic information records through quick response $(\mathrm{QR})$ code and smartphone technology. QR codes have been rapidly applied in medicine (Kobo et al., 2021), veterinary (Chen et al., 2016), food safety (Matzembacher et al., 2018), and animal management (Green et al., 2017; Hu et al., 2020). To enhance the efficiency of sea turtle rescue, the QR code was fused with the turtle carapace, thus saved a large amount of traceability information.

\section{MATERIALS AND METHODS}

Turtle QR code platform was built based on the global positioning technology (GPS), geographic information system (GIS), QR code, Internet network, and mobile terminal. Everyone can scan the QR code by using WeChat to check the turtle information and upload new information to the platform.

It is difficult to print QR code directly on the turtle carapace, because live turtles always move during the $\mathrm{QR}$ code printing process for 1-3 min. Therefore, a fiber laser marking machine (model: YLP-F20, working parameters: speed 2,000 mm/s, power $30 \%$, frequency $20 \mathrm{kHz}$ ) was used to print the QR code on ABS plastic (non-toxic, odorless, and harmless) and then stick it on the turtle carapace. The four sides of the QR code label were indicated by individual number, rescue telephone, release address, and release department in Chinese and English to facilitate international rescue cooperation. After inputting the basic information of the turtle by an administrator, who scanned the QR code using WeChat App, the QR code was pasted on the turtle carapace that has been sanitized, washed, disinfected with alcohol, degreased with acetone, and dried. And then, these turtles were released to the wild.

Once the released turtle is stranded or captured during the fishing operation, the QR code can be scanned using the WeChat app to view the turtle basic information (e.g., age and location) and upload the stranded turtle information (e.g., contact information, time, location, and fishing tools) to the platform. The platform obtained IP positioning, asked for the permission of GPS positioning, and immediately informed the reserve to rescue turtles in time.

\section{Feasibility Test of Sticking Quick Response Code With Epoxy Resin Glue}

A total of ninety, including 30 one-year-old, 30 three-yearold, and 30 eight-year-old green sea turtles were collected from Huidong Sea Turtle National Nature Reserve. Using epoxy resin glue, the QR code was pasted on the carapace that has been sanitized, washed, disinfected with alcohol, degreased with acetone, and dried. The condition of QR code adhesion was monitored every 10 days.

\section{Detection of Different Glue Adhesive on 1-Year-Old Turtles}

Due to the rapid growth and metabolism of young turtles, the durability of QR code glued with epoxy resin glue is poor. Three other types of glue, including acrylic glue (V783, Valigoo, Dongguan Lelai Adhesive Products Co., LTD, Guangdong Province, China), ABS glue (BXS1033, Boshidun, Dongguan Boshidun adhesive Co., LTD, Guangdong Province, China), and nail glue (401, YongZaen, Shenzhen Gusubang Industrial Co., LTD, Guangdong Province, China) were tested on 10 one-year-old green turtles. The QR code was pasted on the carapace with an operation time of $30 \mathrm{~s}$ (acrylic glue), $5 \mathrm{~min}$ (ABS glue), and $40 \mathrm{~s}$ (nail glue). The condition of QR code adhesion was observed and recorded every 10 days.

\section{Application of Quick Response Code in Turtle Release}

On September 8, 2020, 18 eight-year-old green turtles (Table 1) with an average body weight of $26.0 \pm 4.2 \mathrm{~kg}$ were transported to Yanzhou Rewilding Base for rewinding training. On June 1, 
2021, all turtles were marked with PIT chips (HPT9; Biomark, Boise, ID, United States). Among them, 15 turtles were tagged with a steel plate under the left forelimb, and a $\mathrm{QR}$ code was pasted with epoxy resin glue. On June 8, 2021, two turtles (chip no. 900108000628671 and 999001003207185) were fitted with satellite trackers (ID: 210325 and 220639) to learn their movements. Three turtles marked with PIT chips were considered the control group. On June 8, 2021 (World Oceans Day), sea turtles were released to the South China Sea on the beach of Huizhou Turtle Bay. The effect of application of QR code was discussed through the rescue cases of green turtles within 50 days after release.

\section{RESULTS}

\section{Feasibility of Sticking Quick Response Code With Epoxy Resin Glue}

After 50 days of observation and recording, the intact rate of pasted QR code is obtained, as shown in Table 2. The integrity rate of QR code was found to be $100 \%$. The adherence rate of QR code of 3-year-old sea turtles was $73 \%$, while that of 1 -year-old group was only $30 \%$.

\section{Adhesion of Different Glue on 1-Year-Old Turtles}

The nail glue 401 showed the best adhesion effect. The integrity rate of QR code was $100 \%$ after 50 days and reduced to $90 \%$ after
70 days. The QR code pasted with ABS glue fell off quickly, and the integrity rate decreased to $60 \%$ after 10 days and $20 \%$ after 50 days. The QR code pasted with Acrylic glue began to fall off after 30 days, and the integrity rate reduced to $70 \%$ after 50 days.

\section{Application of Quick Response Code in Turtle Released Within $\mathbf{5 0}$ Days}

From June 8 to July 28, 2021, the Huidong Sea Turtle National Nature Reserve handled five turtle rescue cases. The rescue time of the five stranded turtles ranged between 5 and 29 days since they were released to a wide sea. The distance from the released site ranged from 9.5 to $750.85 \mathrm{~km}$. Three of the five cases were reported through the turtle QR code platform (60\%).

The turtle with QR code no. GK000533 was stranded on the beach near the Pinghai sand factory in Xiaoao Bay $(22 \mathrm{~km}$ away from the release site). On June 16, 2021, a turtle without a QR code marker was stranded on the beach of Vanke Double Moon Bay (9.5 km away from the release site). At the time of rescue, it was dead and decayed, and could not read the information in the PIT. On June 26, 2021, the third rescued turtle was stranded on the beach near Pinghai power station (29 $\mathrm{km}$ away from the release site). It was released by a local rescuer without a PIT scanner. The fourth stranded green turtle (QR code: GK000372) mistakenly entered Yuandun River in Shenzhen-Shantou Cooperation Zone and then floated on the water. The fifth stranded turtle with QR code no. GK000395 was found on the Golden Beach of Hainan Lingao Country Garden (750.85 $\mathrm{km}$ away from the release site) and just died when it was

TABLE 1 | Information of released green sea turtles.

\begin{tabular}{|c|c|c|c|c|c|c|}
\hline Serial number & Chip number & QR code number & Steel plate number & Age (year) & Weight (kg) & State of health \\
\hline 1 & 900108000628671 & GK000530 & CHN02076 & 8 & 34.6 & Healthy \\
\hline 2 & 999001003207185 & GK000536 & CHN02079 & 8 & 30.3 & Healthy \\
\hline 3 & 690020037861374 & GK000537 & CHN02077 & 8 & 30.7 & Healthy \\
\hline 4 & 690020037861331 & / & / & 8 & 24.0 & Healthy \\
\hline 5 & 900108000629104 & GK000403 & CHN02095 & 8 & 19.4 & Healthy \\
\hline 6 & 999001003319251 & GK000529 & $\mathrm{CHN} 02081$ & 8 & 22.7 & Healthy \\
\hline 7 & 900108000627735 & GK000378 & CHN02093 & 8 & 22.5 & Healthy \\
\hline 8 & 690201401210602 & GK000523 & CHN0762 & 8 & 26.5 & Healthy \\
\hline 9 & 900108000628719 & GK000528 & CHN02078 & 8 & 29.3 & Healthy \\
\hline 10 & 900108000627816 & GK000533 & CHN02096 & 8 & 28.2 & Healthy \\
\hline 11 & 999001003311546 & GK000526 & CHN02083 & 8 & 30.3 & Healthy \\
\hline 12 & 900108000627788 & GK000395 & CHN02098 & 8 & 22.0 & Healthy \\
\hline 13 & 900108000627852 & GK000534 & CHN02094 & 8 & 21.0 & Healthy \\
\hline 14 & 999001003332526 & GK000372 & CHN02091 & 8 & 21.4 & Healthy \\
\hline 15 & 900108000627856 & GK000538 & CHN02080 & 8 & 29.4 & Healthy \\
\hline 16 & 690020037861300 & GK000524 & CHN02082 & 8 & 24.8 & Healthy \\
\hline 17 & 999001003321029 & / & I & 8 & 25.0 & Healthy \\
\hline 18 & 982091061222713 & / & / & 8 & 27.0 & Healthy \\
\hline TOTAL & 18 & 15 & 15 & 8 & $26.0 \pm 4.2$ & Healthy \\
\hline
\end{tabular}

TABLE 2 | Rate of well-pasted QR code stuck with epoxy resin glue in different age turtles.

\begin{tabular}{|c|c|c|c|c|c|c|}
\hline Turtle Age & Amount & 10 days later & 20 days later & 30 days later & 40 days later & 50 days later \\
\hline One-years-old & 30 & 30 (100\%) & 26 (87\%) & 19 (63\%) & $14(47 \%)$ & 9 (30\%) \\
\hline Three-years-old & 30 & 30 (100\%) & 30 (100\%) & 30 (100\%) & 25 (83\%) & 22 (73\%) \\
\hline Eight-years-old & 30 & 30 (100\%) & 30 (100\%) & 30 (100\%) & 30 (100\%) & $30(100 \%)$ \\
\hline
\end{tabular}


rescued on June 12, 2021. There were no new turtle rescue cases in the following month.

Satellite tracking of turtles equipped with satellite trackers showed that they prefer to migrate along the coastline and could reach the safe wild foraging site within a month (Figure 1). The turtle with ID 220639 took the road to the southwest, crossed Daya Bay, the Outer Lingding Ocean, and Qiongzhou Strait, and finally arrived at Yubone Harbor in Lingao County of Hainan Province on July 7. The turtle with ID 210325 remained active in the waters near Turtle Bay and arrived at Langhua Reef in the Xisha Islands on July 18. And then, the turtles continued to move around.

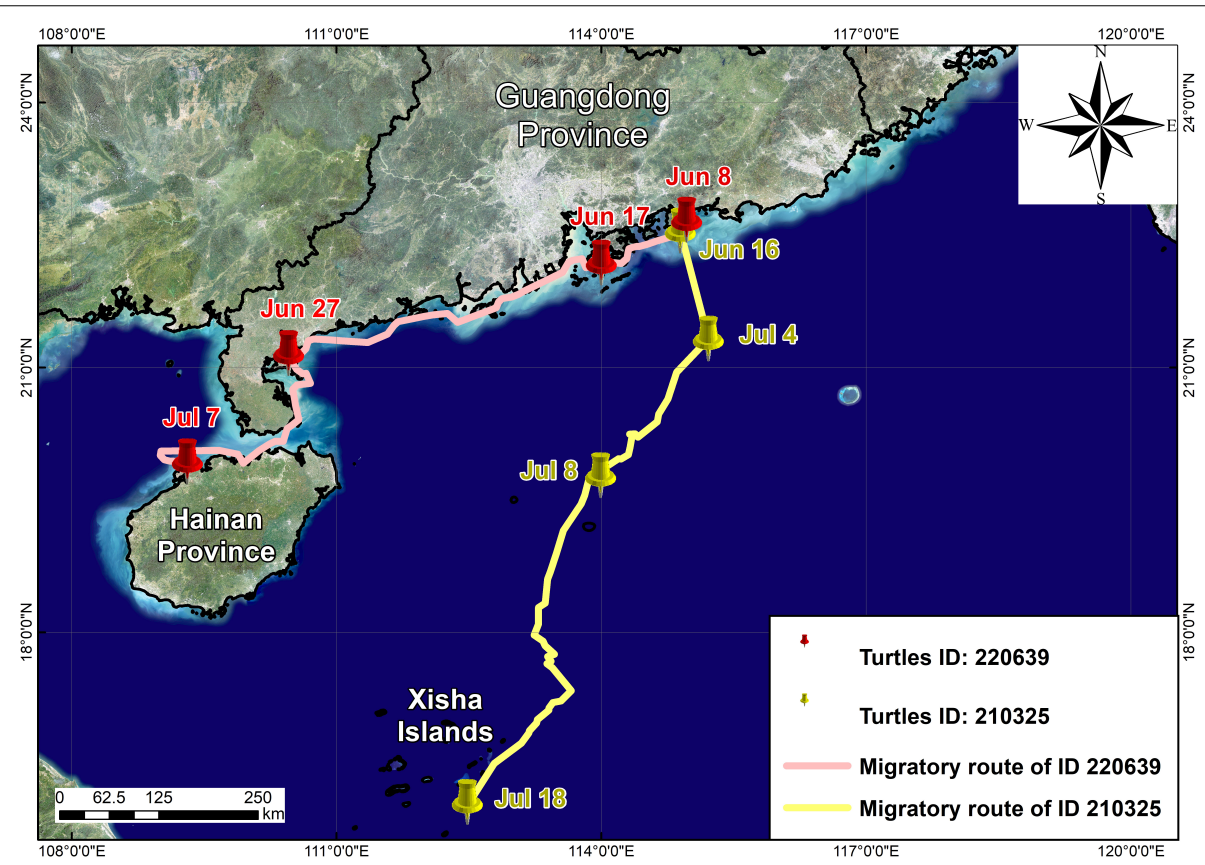

FIGURE 1 | Migratory route of 2 turtles fitted with satellite trackers.

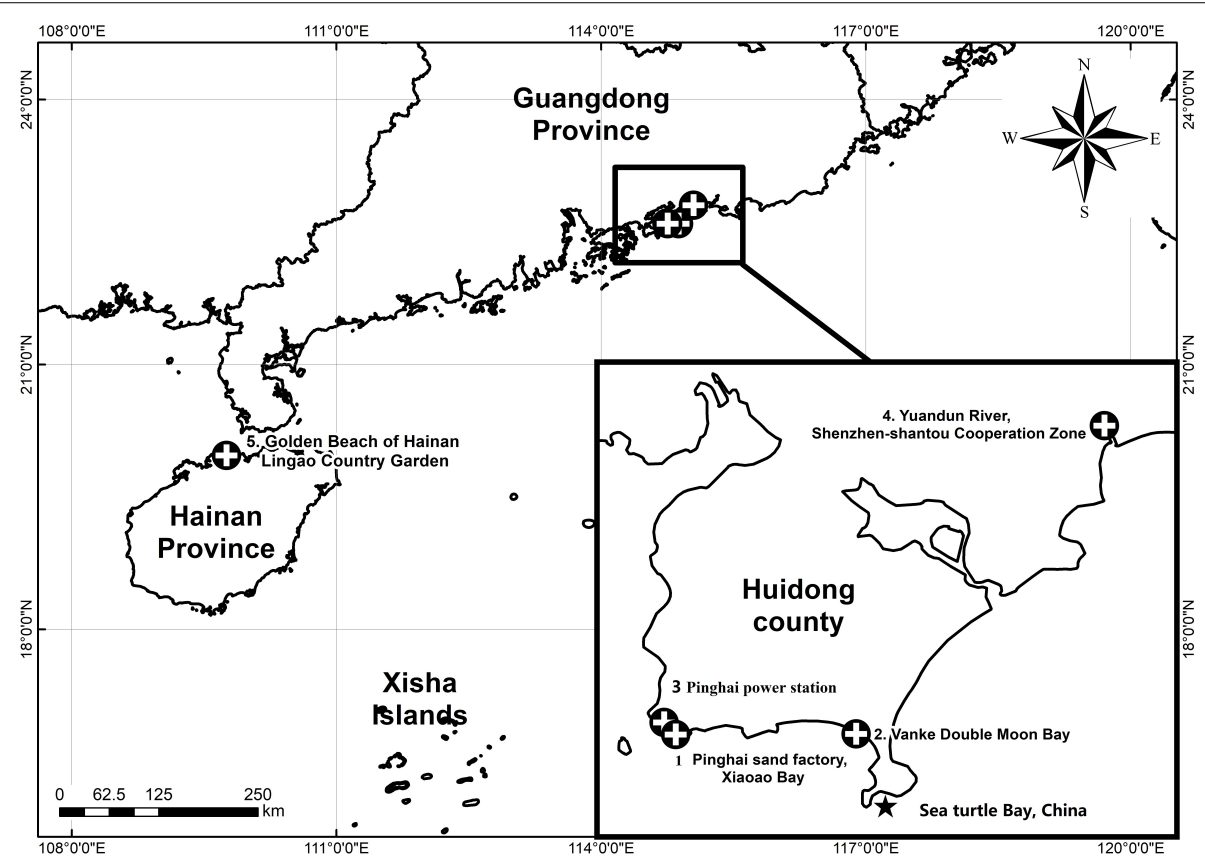

FIGURE 2 | Rescue locations of 5 rescued turtles from June 8 to July 28, 2021. 


\section{DISCUSSION}

\section{Advantages of Quick Response Code as a Turtle Marker}

Passive integrated transponder tags are commonly used as individual identification markers for sea turtles management. PIT tags are relatively small, injectable, and durable and can be read remotely without viewing the tag. However, only researchers in nature reserves or university research institutes, and few fishermen or fishery law enforcement agencies are equipped with such scanning tools, thus reducing the use of PIT chips. Steel plate is the representative of traditional tags, which are intuitive and simple, but easy to fall off and lose. Since QR code has been widely used and recognized in many industries, we try to apply it to the individual identification and rescue of released turtles. At present, smartphones are very popular, QR codes are easy to popularize and show a high degree of interactivity in a wide range of application. The QR code is directly scanned by mobile phone to facilitate public participation in rescue and management. To overcome the limitation of a single label, we tried to use the joint marking method of steel plate, PIT chip, and QR code to make up for the deficiency of a single label. When used together, they are complementary to one another, which is conducive to grasping the information of individual identification of the rescue turtles to the maximum extent and improving the rescue efficiency.

The application of turtle QR code platform in the released turtle showed that the QR tag is a powerful alternative to other markers. It improves rescue efficiency by realizing the interaction between the public and the turtle rescue personnel. Through the information reported by the $\mathrm{QR}$ code, the rescue staff of reserve can rescue turtles in time. Even if the turtle is dead, zoological researchers can collect samples from fresh carcasses for endangered species conservation studies, such as the turtle in the first and fifth cases. In the fourth stranded case, the green turtle (QR code: GK000372) floated on the water due to osmotic imbalance. The discoverer scanned the QR code and contacted the Huidong Sea Turtle National Nature Reserve in time. After 2 days of care under the guidance of the rescue staff, the turtle was released once again on June 29 at the seaside of Paijiao, Shanwei, China. However, the stranded turtles with PIT chips or steel tags are often rotten and smelly due to the lack of timely rescue.

The first month after release is critical to the success of release, as it takes about a month for released individuals to reach wild foraging sites (Figure 1). Epoxy resin glue has the best adhesion effect on 8-year-old turtles, and the intact rate after 50 days was $100 \%$. Therefore, the QR code pasted by epoxy resin glue can be used for the rescue of released turtles when it was stranded within 50 days after release. The application of pollution-free QR code technology in animal management can improve management efficiency, provide evidence for criminal investigation, and play a role in combating the illegal trade of wildlife (Visser and Hanich, 2018). The adhesion effect of nail gel 401 on the back nail of 1 year-old turtles after 50 days is the best (100\%), which can be used for short-term conservation of young turtles.

The QR code information platform is utilized to mobilize the enthusiasm and participation of fishermen and enthusiasts in the conservation of sea turtles and continuously evaluate the distribution pattern, change trend, and existing problems of turtle population, to promote the recovery of endangered species in the world.

\section{Main Reason of Stranding Turtles Released From the Turtle Bay, China}

Huidong Sea Turtle National Nature Reserve is the only turtle nursery in China. In 2017, the key technology of artificial breeding of green turtles was developed, and thousands of young turtles are produced every year for breeding, releasing, or popularizing science. Satellite tracking of previously released turtles showed that they prefer to migrate along coastlines for food (Xia and Gu, 2012), as did the five rescued turtles (Figure 2) and two turtles equipped with satellite trackers (Figure 1) in this study. The migration distance of the five turtles ranged from 9.5 to $750.85 \mathrm{~km}$, and the dispersal rate ranged from 1.05 to $25.89 \mathrm{~km} /$ day. The places where turtles are stranded or lost are often power stations, beaches, or bays with human disturbance, pollution, or fishery prosperity.

In this study, the stranding of four turtles had been linked to pollution. Two turtles are stranded in Xiaoao Bay, which is about $500 \mathrm{~m}$ deep with the narrowest distance of the entrance of only $185 \mathrm{~m}$, making it easy to get in and hard to get out. Cooling water discharged from the Pinghai power station of Xiaoao Bay attracted the turtles because it was warmer than the surrounding sea; however, the warmer water contained a variety of pollutants, increasing the risk of stranding. The highly polluted seawater was caused by many seascape houses and tourists near the beach of the Vanke Double Moon Bay. The water velocity of Golden Beach of Hainan Lingao Country Garden near Qiongzhou Strait is rapid and the water is highly polluted. The last turtle stranded in Yuandun River was thought to be caused by the high permeable pressure of freshwater.

\section{CONCLUSION}

Quick response code is a powerful measure to overcome the limitations of the existing individual label in the conservation of released turtles. The QR code information platform is utilized to mobilize the enthusiasm and participation of fishermen and enthusiasts in the conservation of sea turtles. However, the QR code fused with turtle carapace can only be applied for turtle rescue and tracing in the short-term due to its poor persistence.

\section{DATA AVAILABILITY STATEMENT}

The original contributions presented in the study are included in the article/supplementary material, further inquiries can be directed to the corresponding author/s.

\section{AUTHOR CONTRIBUTIONS}

DY and HH conceived the study and drafted the manuscript. ZX carried out the field work and contributed to breed turtles. HY participated in the data analysis. FY and XL contributed to release turtles. All authors approved the manuscript for publication. 


\section{FUNDING}

This study was supported by the Science and Technology Innovation Project of Guangdong Forestry (2020KJCX005), Special Marine Projects (Animal diversity monitoring),

\section{REFERENCES}

Chen, T. S., Chen, T. L., Chung, Y. F., Huang, Y. M., Chen, T. C., Wang, H., et al. (2016). Implementation of online veterinary hospital on cloud platform. J. Med. Syst. 40:147. doi: 10.1007/s10916-016-0501-x

Green, T., Smith, T., Hodges, R., and Fry, W. M. (2017). A simple and inexpensive way to document simple husbandry in animal care facilities using QR code scanning. Lab. Anim. 51, 656-659. doi: 10.1177/0023677217718004

Hu, Y. Y., Tao, Y. H., and Kong, S. S. (2020). Introduction of a web-based novel laboratory animal management system. Labor. Animal Comp. Med. 40:261. doi: 10.1177/10406387211003163

Katalinas, C. J., Brenkert, K., Darden, T., and Denson, M. R. (2018). A genetic assessment of a red drum, sciaenops ocellatus, stock enhancement program. J. World. Aquacult. Soc. 49, 523-539.

Kitada, S. (2018). Economic, ecological and genetic impacts of marine stock enhancement and sea ranching: a systematic review. Fish. Fish. 19, 511-532. doi: $10.1111 /$ faf. 12271

Kitada, S. (2020). Lessons from Japan marine stock enhancement and sea ranching programmes over 100 years. Rev. Aquacult. 12, 1944-1969.

Kobo, O., Contractor, T., Mohamed, M. O., Parwani, P., Paul, T. K., Ghosh, R. K., et al. (2021). Impact of pre-existent vascular and poly-vascular disease on acute myocardial infarction management and outcomes: an analysis of 2 million patients from the national inpatient sample. Int. J. Cardiol. 327, 1-8. doi: 10.1016/j.ijcard.2020.11.051

Loneragan, N. R., Taylor, M. D., and Tweedley, J. (2019). A drop in the ocean: marine fish releases in Australia. Austral. Ecol. 44, 545-551. doi: 10.1111/aec. 12722

Matzembacher, D. E., Stangherlin, I. D. C., and Slongo, L. A. (2018). An integration of traceability elements and their impact in consumer's trust. Food. Control. 92, 420-429. doi: 10.1016/j.foodcont.2018.05.014
Science and Technology Planning Project of Huizhou, Guangdong Province, China (No. 2020SC0302019), Science and Technology Planning Project of Guangdong Province, China (2019B121202004), and GDAS Special Project of Science and Technology Development (2018GDASCX-0107).

Ueda, H. (2018). "Migration and navigation in fish," in Encyclopedia of Reproduction, ed. M. K. Skinner (Oxford: Academic Press).

Vilaça, S. T., Piccinno, R., Rota-Stabelli, O., Gabrielli, M., Benazzo, A., Matschiner, M., et al. (2021). Divergence and hybridization in sea turtles: inferences from genome data show evidence of ancient gene flow between species. Mol. Ecol. 30, 6178-6192. doi: 10.1111/mec.16113

Visser, C., and Hanich, Q. A. (2018). How blockchain is strengthening tuna traceability to combat illegal fishing. Conversation 22, 1-4.

Xia, Z. R., and Gu, H. X. (2012). Reports of satellite tracking green sea turtles in china. Sichuan J. Zool. 31, 435-438.

Conflict of Interest: FY was employed by the company Xiyan Natural Resources Technology (Guangzhou) Co., Ltd.

The remaining authors declare that the research was conducted in the absence of any commercial or financial relationships that could be construed as a potential conflict of interest.

Publisher's Note: All claims expressed in this article are solely those of the authors and do not necessarily represent those of their affiliated organizations, or those of the publisher, the editors and the reviewers. Any product that may be evaluated in this article, or claim that may be made by its manufacturer, is not guaranteed or endorsed by the publisher.

Copyright (c) $2022 \mathrm{Xia}, \mathrm{Yu}, \mathrm{Yang}, \mathrm{Liu}, \mathrm{Yu}$ and Hu. This is an open-access article distributed under the terms of the Creative Commons Attribution License (CC BY). The use, distribution or reproduction in other forums is permitted, provided the original author(s) and the copyright owner(s) are credited and that the original publication in this journal is cited, in accordance with accepted academic practice. No use, distribution or reproduction is permitted which does not comply with these terms. 